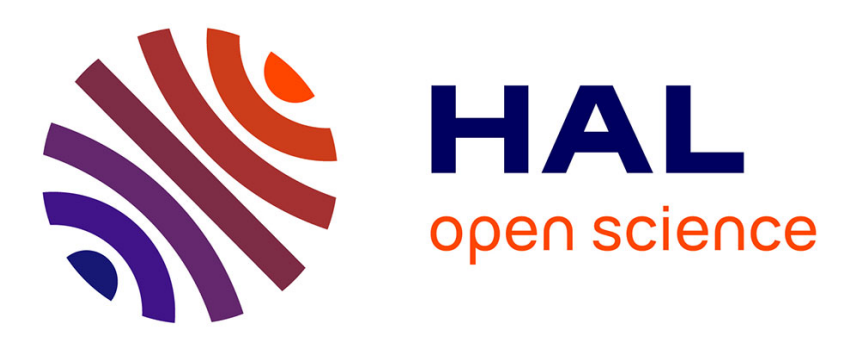

\title{
Doubly-resonant SFG and DFG spectroscopies: an analytic model for data analysis including distorted and rotated vibronic levels. I. Theory
}

Bertrand Busson

\section{- To cite this version:}

Bertrand Busson. Doubly-resonant SFG and DFG spectroscopies: an analytic model for data analysis including distorted and rotated vibronic levels. I. Theory. Journal of Chemical Physics, 2020, 153 (17), pp.174701. 10.1063/5.0022760 . hal-02935624

\section{HAL Id: hal-02935624 \\ https://hal.science/hal-02935624}

Submitted on 10 Sep 2020

HAL is a multi-disciplinary open access archive for the deposit and dissemination of scientific research documents, whether they are published or not. The documents may come from teaching and research institutions in France or abroad, or from public or private research centers.
L'archive ouverte pluridisciplinaire HAL, est destinée au dépôt et à la diffusion de documents scientifiques de niveau recherche, publiés ou non, émanant des établissements d'enseignement et de recherche français ou étrangers, des laboratoires publics ou privés. 
Doubly-resonant SFG and DFG spectroscopies: an analytic model for data analysis including distorted and rotated vibronic levels. I. Theory

\author{
Bertrand Busson ${ }^{1}$ \\ Université Paris-Saclay, CNRS, Institut de Chimie Physique, UMR 8000, \\ 91405 ORSAY, France ${ }^{\text {a) }}$
}

(Dated: 10 September 2020)

We present an analytic description of doubly resonant infrared-visible sum (SFG) and difference frequency generation (DFG) spectroscopies. Within the BornOppenheimer and Condon approximations for harmonic oscillators, we extend the usual theory, limited to linear electron-vibration coupling, and introduce the quadratic coupling phenomena (mode distortion and mode mixing) in the excited state. The excitation spectra of vibrations in SFG and DFG experiments are calculated in integral form for arbitrary mode distortions and small amplitude mode mixing between pairs of modes. Mode distortion modifies all orders of vibronic coupling including the fundamental process, whereas mode mixing appears as a perturbation added to the distorted mode case. For small quadratic coupling amplitudes, the results may be recast in simple analytic forms after introduction of the overlap spectral function, and developed in sums and products of Lorentzian functions.

\footnotetext{
a)Electronic mail: bertrand.busson@universite-paris-saclay.fr
} 


\section{INTRODUCTION}

For the past years, infrared-visible Sum Frequency Generation (SFG) vibrational spectroscopy, and to a lesser extent its counterpart Difference Frequency Generation (DFG) spectroscopy, ${ }^{1,2}$ have become widely used investigation techniques for the chemical analysis of interfaces. ${ }^{3}$ Due to the symmetry properties of second order nonlinear optics, such frequency generation (FG) processes are intrinsically surface and interface specific. As vibrational spectroscopies, they become singly resonant (SR) with vibrational transitions in the infrared (IR) to resonantly probe various kinds of interfaces: molecules at solid surfaces in vacuum, ${ }^{4}$ in air, ${ }^{5}$ in catalytic conditions ${ }^{6}$ or under electrochemical control $;{ }^{7}$ free, ${ }^{8}$ buried $^{9}$ or monolayer covered ${ }^{10}$ surfaces of liquids; nanostructured materials. ${ }^{11,12}$ As for all SR IR processes (IR absorption, Raman scattering, SR-FG), the molecular response leads to rather handy expressions with a Lorentzian description of the vibration modes, ${ }^{13}$ the activity of each mode depending only on its own vibrational parameters (mode separability). The experimental FG resonant response, modelled by Lorentzian functions, is then carefully ${ }^{14}$ fitted by a numerical adjustment of the free parameters for a fast result (although alternates exist $^{15,16}$ ). When the visible or generated FG beams match the energy and selection rules of an allowed electronic transition, the processes becomes doubly resonant (DR), ${ }^{17}$ further enhancing the measured signals through molecular amplification. DR processes also open the opportunity to probe the molecular electronic and vibronic transitions at interfaces by using a two-color experimental set-up, that is with independently tunable (or broadband) IR and visible lasers. ${ }^{18}$ Excitation of molecular electronic resonances in the FG processes implies to take into account the contributions of the whole vibrational structure of the excited state (vibronic states), in other words there is no vibronic mode separability. This introduces additional difficulties in experimental procedures and data analysis, which may account for the low use of DR-FG spectroscopy so far in spite of its obvious interest. However, after the promising first experimental results, ${ }^{19-23}$ literature has recently shown a renewed interest to exploit the specificities of DR-SFG. ${ }^{24-34}$

Theory for doubly resonant FG processes follows the formalism developed for resonant Raman scattering (RRS). The basic models rely on standard (Born-Oppenheimer, harmonic vibrations, Condon and/or Herzberg-Teller) approximations, and the level of accuracy of the vibronic structure description governs the complexity of the model. The Hamiltonian 
describing the electronic excited state may involve linear and quadratic electron-vibration coupling terms. In the linear case, the vibronic structure is built on the same vibrational levels as in the ground state, except for displacements in the positions of the harmonic potential curves along the normal mode coordinates (Figure 1), vanishing for non-totally symmetric modes. ${ }^{35}$ It can be shown ${ }^{36,37}$ that quadratic coupling is responsible for vibrational frequency shifts (distortion of the harmonic potentials) and mode mixing (Duschinsky rotation) in the excited state. Apart from two papers from Vallet et al., ${ }^{38,39}$ the influence of mode distortion and mode mixing has never been investigated in DR-FG processes. On the contrary, an abundant literature theoretically describes the excitation of vibronic transitions by light-matter interaction in RRS phenomena, and we may essentially divide it in three methods: the sum-over-states (SOS) procedure, the transform technique (TT) and the time-dependent method (TDM).

The SOS method explicitly calculates the Franck-Condon overlap integrals ${ }^{40,41}$ and sums up all vibronic contributions. It leads to a description as sums and products of Lorentzian functions, well adapted to experimental data fitting, but makes it necessary to completely define each vibration mode in the lower and upper electronic states and involves large sums over vibrational quanta.

The transform technique ${ }^{42,43}$ was originally aiming at getting rid of these difficulties by embedding the SOS calculations into quantities directly extracted from experimental data (e.g. absorption, luminescence, fluorescence). Through the time correlator formalism, TT allows to separate the response of each vibration mode while embedding the contributions of all other modes of the vibronic structure into the overlap spectral function $\Phi(\omega)$, closely related to the correlator building up the absorption spectrum. ${ }^{44}$ Unfortunately, the Raman time correlator cannot be factorized by the absorption one and must be evaluated by orders. ${ }^{39}$ This method does not allow a direct fitting of the experimental data to extract parameters related to the vibronic levels. Additionally, the formalism becomes rather recondite and this may reveal disheartening for a quick spectroscopic data analysis.

Identically, the basic idea of the time-dependent model is to transform the SOS into a time dependent correlator. The difference with TT lies in the subsequent step: emphasis is put on the wavefunctions rather than on the Hamiltonian. The time correlator is expressed as the evolution of a time dependent wavepacket, ${ }^{45}$ for which a Gaussian form is assumed from the harmonic natures of the potentials. ${ }^{46,47}$ Considering that only short times matter 
in a Raman process, a semi classical propagation dynamics may be applied. ${ }^{45}$ This leads to an exact calculation of the Raman intensities, without any separation into orders. For this reason, this formalism appears more powerful than the two others as far as RRS is concerned. However TDM may not apply to very large molecules, ${ }^{47}$ is limited to vanishing temperatures as only the ground state is considered ${ }^{48}$ and seems to have been less extensively studied in the literature. ${ }^{49}$

The linear coupling model imperfectly accounts for the real behavior of many molecular systems, in particular large organic molecules, for which distortion and mode mixing are far from being uncommon. ${ }^{50}$ The TT and TDM formalisms allow to elegantly integrate the effects beyond the linear coupling and Franck-Condon approximations. Still, it is possible to perform some SOS integration of Franck-Condon factors in the quadratic coupling scheme under some approximations (low temperature, small frequency shifts, small quadratic coupling) by introducing the hypotheses of mode mixing ${ }^{36,41,51-53}$ and mode distortion ${ }^{40,54}$ into the model. A direct SOS calculation including non-Condon terms has also been reported. ${ }^{55}$ For the most studied TT method, it has been shown possible to introduce in the RRS models of vibronic structure: quadratic electron-vibration coupling, ${ }^{56,57}$ non-Condon terms, ${ }^{58,59}$ inhomogeneous broadening ${ }^{60}$ anharmonicity in Morse potentials,${ }^{61}$ temperature dependence, ${ }^{44}$ breaking of the $\mathrm{BO}$ approximation ${ }^{62}$ and combinations of these effects. ${ }^{42,63,64} \mathrm{TDM}$ has the additional advantage to keep the Hamiltonian hidden in the time dependent wavepacket, calculated separately. Non-Condon terms may therefore be introduced into the theory provided that the purely Gaussian wavefunctions are replaced by a decomposition over Gaussian basis sets, for which the propagation may be calculated. ${ }^{46,65}$ Mode mixing and frequency shifts are introduced by hand into the theory, through their effects on the vibrational wavefunctions, and at the cost of increasing complexity. ${ }^{66,67}$

By analogy to RRS, all three approaches were applied to DR-FG in the linear coupling case, with the great difference that the SFG time-correlator may be explicitly calculated without separating into orders. ${ }^{39}$ As early as 1994, the first time correlator model was established for DR-SFG and DR-DFG ${ }^{68}$ within the same original frame as for RRS, with a result very similar to SR-SFG coupled to a heat bath. ${ }^{13}$ To our knowledge, the transform idea (i.e. to evaluate the vibronic contribution from experimental absorption data through the overlap spectral function) has never been applied to the analysis of experimental DRFG data. SFG usually applies to monolayers on solid or liquid substrates, for which the 
quantity of matter makes it difficult, albeit not impossible, to experimentally measure a high resolution absorption spectrum in situ. In fact, the TT formulation comes down to a sum-over-states with usual energy denominators by expanding the exponentials of the time correlators into orders for a generic vibration mode, and integrating over time. The reverse transformation (SOS into TT) is evidenced in the derivation of Ref. 69 which expresses the result of the SOS approach as products of time correlators. As a consequence, all molecular approaches of DR-FG processes converge to a common formalism which can be introduced either through the conventional SOS method ${ }^{69}$ or by a time correlator in a TT or TDM formulation. ${ }^{70}$ This derivation forms the basis of conventional interpretation of DR-FG data in the literature. ${ }^{20,22,23}$

To a lesser extent than for RRS, extensions have been introduced in DR-FG beyond the conventional approximations. The main approaches focus on the inclusion of nonCondon $^{28,70}$ and non-adiabatic ${ }^{71,72}$ terms, in particular to account for the specific properties of DR-SFG from chiral liquids. As for quadratic coupling, one formulation of the DR-SFG response including mode distortion and mode mixing in the TT frame has been published. ${ }^{38,39}$ However, it has never been applied to study experimental data because the TT formalism forbids a direct procedure for data fitting (there is no straightforward mode separation into energy resonant Lorentzians) and accurate parameters describing molecular vibronic structure still lack. As for this second reason, the constant improvement of computational chemistry over the past decades makes this limitation become obsolete. It now allows to calculate numerous molecular parameters on bigger systems, including Franck-Condon factors, mode distortions and Duschinsky matrices. In fact, the continuous improvement in computation of molecular electronic transitions can be traced through the simulations of RRS and DR-SFG data. RRS spectra in particular have been simulated using HartreeFock, ${ }^{73,74} \mathrm{CASSCF},{ }^{52}$ configuration interaction, ${ }^{50,66,75,76} \mathrm{MP}$ perturbation theory ${ }^{50}$ and more recently (TD-)DFT ${ }^{50,66,77-82}$ in the linear coupling scheme. Introduction of non-Condon ${ }^{83}$ and quadratic coupling terms ${ }^{84}$ follows naturally, and a methodology for a complete vibronic structure calculation by first principle methods is now available. ${ }^{48}$ Calculation of DR-SFG spectra by configuration interaction ${ }^{85}$, DFT/TD-DFT ${ }^{70}$ or $\mathrm{DFT} / \mathrm{CCSD}^{27}$ have appeared more recently but start becoming a routine way to analyze experimental data.

To date, there still lacks a formalism to actually analyze DR-FG data within the quadratic coupling scheme. In this paper, we propose to build on the existing theories (Hayashi et al. ${ }^{69}$ 
for linear coupling and Vallet et $a l .{ }^{39}$ for quadratic coupling) to generate explicit analytical relationships useful for effective data analysis in the small quadratic coupling case. Our goal is to provide DR-FG users with functions usable for actual fitting and quantitative analysis of experimental data beyond the linear coupling approximation. The results are recast into compact formulations through the $\Phi$ function, ${ }^{68}$ which allows to perform the sum-over-states calculations only once, or to extract it from absorption data in the spirit of the transform technique.

The paper is organized as follows: in part II, we review the key notions and quantities used to model the DR-SFG and DR-DFG responses, the vibronic structure and the overlap spectral $\Phi$ function. We review the calculation of $\Phi^{L C}(\omega)$ along with DR-SFG and DRDFG hyperpolarizabilities in the linear electron-vibration coupling scheme. In part III, we introduce vibronic mode distortions without mode mixing, and show the equivalence of our formalism to the transform technique in that specific case. For low mode distortion, explicit formulas are found for the DR-FG hyperpolarisabilities using the linear coupling overlap spectral function including frequency shifts $\left(\Phi^{F S}\right)$. In part IV, we apply the transform technique formulation to low amplitude mode mixing between two (or an arbitrary number of couples of) vibration modes, and describe mode mixing as a perturbation of the linear coupling case by use of the $\Phi^{F S}$ function.

\section{DR-SFG AND DR-DFG FOR LINEAR ELECTRON-VIBRATION COUPLING}

The general formulas for the vibrational SFG and DFG hyperpolarisabilities are recalled in Appendix A. We suppose the existence of a molecular electronic transition in the energy range probed by the visible and SFG beams, described by its frequency $\left(\omega_{e g}\right.$ or $\left.\omega_{e g}^{0}\right)$ and width $\Gamma_{e g}$, supposed here unique for the whole vibronic structure (even if this point may be debated $\left.^{22}\right)$. Transition energy $\hbar \omega_{e g}$ is defined as the energy required to vertically jump to the upper state from the equilibrium position in the ground state, whereas $\hbar \omega_{e g}^{0}$ quantifies the 0-0 line of the electronic transition ${ }^{58,86}$ (Figure 1). The potential energy surfaces of the ground and excited states are displaced with respect to each other along the normal coordinate of mode $j$ by the shift $\Delta_{j}$ (in normal coordinate units). Some authors ${ }^{77}$ prefer

to use dimensionless displacements $\bar{\Delta}_{j}=\Delta_{j} \sqrt{\omega_{j}^{g} / \hbar}$. The relationship between $\omega_{e g}$ and $\omega_{e g}^{0}$ 

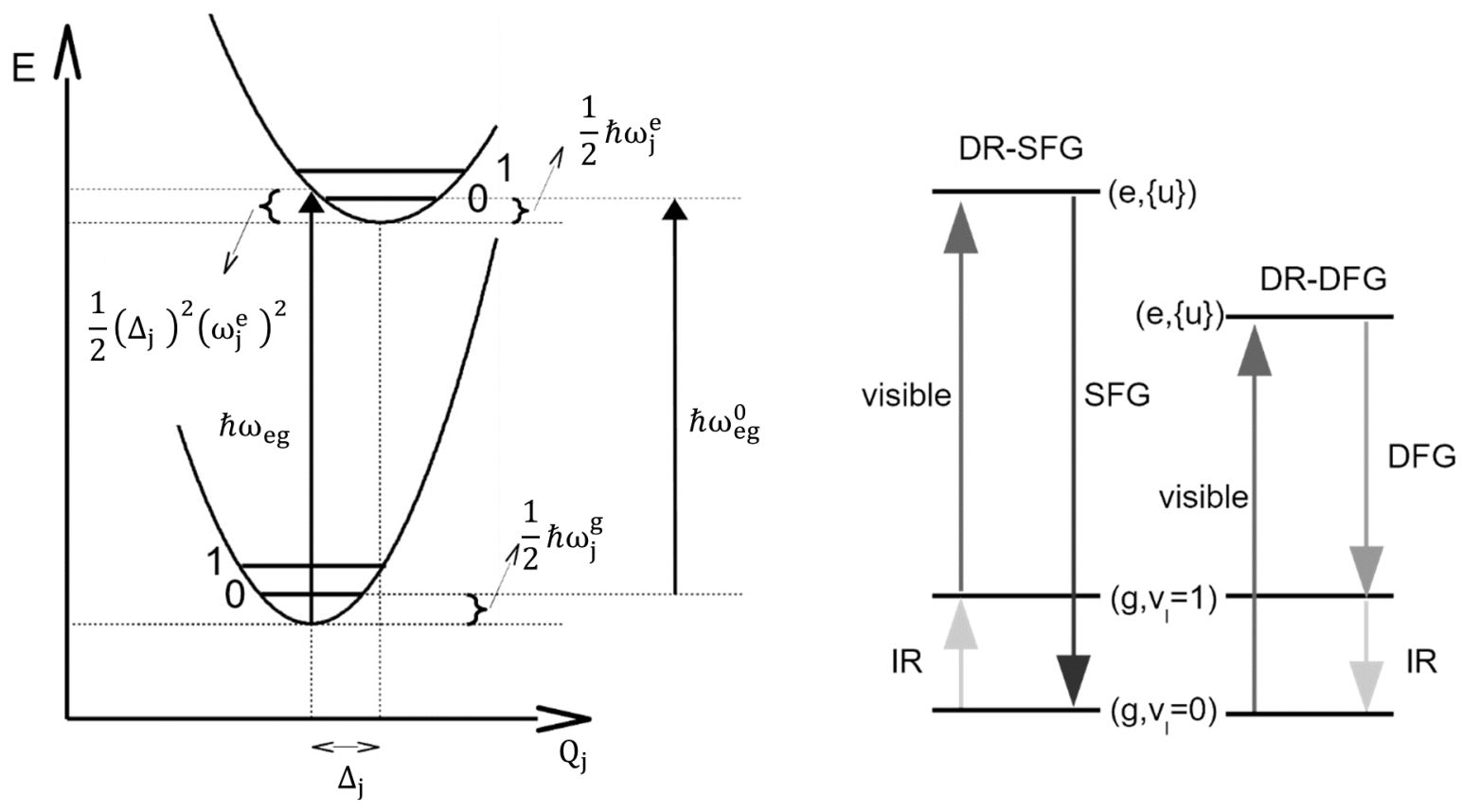

FIG. 1. Definitions of the energies describing the vibrational, electronic and vibronic molecular states. Energetic schemes of the DR-FG processes, with the molecular states involved.

follows from their definitions:

$$
\omega_{e g}^{0}=\omega_{e g}-\sum_{j=1}^{N} \frac{1}{2}\left(\omega_{j}^{g}-\omega_{j}^{e}\right)-\frac{1}{2} \sum_{j=1}^{N} \frac{\left(\omega_{j}^{e} \Delta_{j}\right)^{2}}{\hbar}
$$

where we formally distinguish ground state vibration frequencies $\omega_{l}^{g}$ (and normal mode coordinates $Q_{l}^{g}$ ) from $\omega_{l}^{e}$ (and $Q_{l}^{e}$ ) in the excited state (they are equal under the linear electron-vibration coupling hypothesis) to account for mode distortions introduced in the following parts.

In general, we number $l$ the IR-active mode involved in the vibrational transition of the ground state corresponding to the exchange of one IR photon, and $j$ any generic mode of the vibrational (and vibronic) structure (including mode $l$ ). As SFG and DFG seldom probe modes below $400 \mathrm{~cm}^{-1}$, we may neglect thermal effects and consider that temperature $\mathrm{T}=0 \mathrm{~K}$. Starting from the general equations A5 and A6, the difficulty lies in the SOS calculation over $\{u\}$, and Hayashi et al. ${ }^{69}$ have shown that

$$
\beta_{i j k}^{S F G}=1 / \hbar^{2} \sum_{l}^{I R-a c t i v e} \frac{A_{l}^{i j k} D_{l}^{L C}\left(\omega_{S F G}\right)}{\omega_{I R}-\omega_{l}^{g}+i \Gamma_{l}^{g}}
$$


In the same way,

$$
\beta_{i j k}^{D F G}=1 / \hbar^{2} \sum_{l}^{I R-a c t i v e} \frac{A_{l}^{i j k} D_{l}^{L C}\left(\omega_{D F G}+\omega_{l}^{g}\right)}{\omega_{I R}-\omega_{l}^{g}-i \Gamma_{l}^{g}}
$$

where $A_{l}^{i j k}=\mu_{g \leftarrow e}^{0, i} \mu_{e \leftarrow g}^{0, j}\left(\frac{\partial \mu^{k}}{\partial Q_{l}^{g}}\right)_{Q_{l}^{g}=0}$ is the static FG amplitude of mode $l$, i.e. involving the infrared and electronic activities. Superscript LC refers to the quantities calculated under the linear coupling hypothesis. The excitation spectrum of mode $l, D_{l}^{L C}(\omega)$, encompasses the vibronic transitions resonant with the FG photons after excitation of mode $l$ by the IR photon. We have (details of the calculations may be adapted from Appendix B):

$$
D_{l}^{L C}(\omega)=\frac{1}{i} \int_{0}^{\infty} d t e^{i t\left(\omega-\omega_{e g}^{0}+i \Gamma_{e g}\right)} \tilde{g}_{l}^{L C}(t) \prod_{\substack{j=1 \\ j \neq l}}^{N} g_{j}^{L C}(t)
$$

with

$$
\begin{gathered}
g_{j}^{L C}(t)=e^{2 S_{j} h_{j}^{L C}(t)} \\
\tilde{g}_{l}^{L C}(t)=\Delta_{l} h_{l}^{L C}(t) g_{l}^{L C}(t) \\
h_{j}^{L C}(t)=-\frac{1}{2}\left(1-e^{-i \omega_{j}^{e} t}\right)
\end{gathered}
$$

The quantity

$$
S_{j}=\frac{\omega_{j}^{g}\left(\Delta_{j}\right)^{2}}{2 \hbar}=\frac{\left(\bar{\Delta}_{j}\right)^{2}}{2}
$$

is the Huang-Rhys factor ${ }^{87}$.

In the transform technique, the link between the absorption spectrum and the vibronic structure is efficiently made through function $\Phi(\omega)^{43}$, which sums up the contributions of the vibronic states to the optical transitions. It may be defined in a general manner for an arbitrary temperature as ${ }^{56,88}$

$$
\Phi(\omega)=i \int_{0}^{\infty} e^{i \omega t-\Gamma_{e g} t} \eta(t) d t
$$

where $\eta(t)$ is the optical absorption correlator

$$
\eta(t)=\left\langle\exp \left(\frac{i H_{g} t}{\hbar}\right) \exp \left(\frac{-i H_{e} t}{\hbar}\right)\right\rangle
$$

In the definition of $\eta(t)$, the bracket denotes thermal averaging and projection onto the vibration modes, whereas $H_{g}$ and $H_{e}$ represent the vibrational hamiltonians for the ground 
and excited states, respectively. $\Phi(\omega)$ may be seen as the Fourier transform of the overlap between the vibrational wavefunctions just before and at an arbitrary time after the electronic transition, hence the name (vibrational) overlap spectral function. It can be $\operatorname{shown}^{42,86}$ that the absorption spectrum is expressed as $A(\omega) \propto \omega\left|\mu_{e \leftarrow g}^{0}\right|^{2} \operatorname{Im}[\Phi(\omega)]$, and $\Phi(\omega)$ is sometimes called the complex susceptibility. ${ }^{64}$ Under the linear coupling hypothesis, within our formalism:

$$
\Phi^{L C}(\omega)=i \int_{0}^{\infty} d t e^{i t\left(\omega-\omega_{e g}^{0}+i \Gamma_{e g}\right)} \prod_{j=1}^{N} g_{j}^{L C}(t)
$$

The DR-SFG hyperpolarizability may also be calculated directly using the transform technique in the linear coupling case. ${ }^{68}$ Introduction of function $\Phi^{L C}(\omega)$ is then straightforward, and Eq. 4 can be recast into the form

$$
D_{l}^{L C}(\omega)=\frac{\Delta_{l}}{2}\left[\Phi^{L C}(\omega)-\Phi^{L C}\left(\omega-\omega_{l}^{e}\right)\right]
$$

The great advantage of these expressions is that the overlap spectral function $\Phi^{L C}$ is unique and common to all modes for a given vibronic structure. In other words, it has to be evaluated only once in order to determine the excitation spectra of all vibration modes $l$. Two methods may be chosen for the evaluation of $\Phi$ : either an experimental determination (e.g. from an absorption spectrum), or explicitly by expanding the exponential terms in $g_{j}$ and integrating over time. ${ }^{69}$ It therefore appears at a given order of development as a sum of Lorentzian functions:

$$
\Phi^{L C}(\omega)=e^{-S} \sum_{\substack{k_{1}=0 \\(\ldots) \\ k_{N}=0}}^{\infty}\left(\prod_{j=1}^{N} \frac{\left(S_{j}\right)^{k_{j}}}{k_{j} !}\right) \frac{(-1)}{\omega-\omega_{e g}^{0}-\sum_{j=1}^{N} k_{j} \omega_{j}^{e}+i \Gamma_{e g}}
$$

with $S=\sum_{j=1}^{N} S_{j}$ and, at this stage, $\omega_{j}^{e}=\omega_{j}^{g}$. As an illustration of the use of this function, Eq. 11 may be rewritten as:

$$
\begin{aligned}
D_{l}^{L C}(\omega)=-\frac{\Delta_{l}}{2} e^{-S} \sum_{\substack{k_{1}=0 \\
(\ldots) \\
k_{N}=0}}^{\infty}\left(\prod_{j=1}^{N} \frac{\left(S_{j}\right)^{k_{j}}}{k_{j} !}\right) & {\left[\frac{1}{\omega-\omega_{e g}^{0}-\sum_{j=1}^{N} k_{j} \omega_{j}^{e}+i \Gamma_{e g}}\right.} \\
& \left.-\frac{1}{\omega-\omega_{e g}^{0}-\omega_{l}^{e}-\sum_{j=1}^{N} k_{j} \omega_{j}^{e}+i \Gamma_{e g}}\right]
\end{aligned}
$$


Applying the above formulas, even in the simplest linear coupling case, requires to consider all the vibration modes in the vibronic structure. The Franck-Condon scheme is not very restrictive indeed as far as selection rules are concerned. As pointed out for example in Ref. 62 , all vibronic modes with nonvanishing displacements have an influence on the resonant (RRS or DR-FG) amplitudes of a given ground state vibration mode and must therefore be explicitly taken into account. This is in fact not always the case in the literature, where a truncation of Eq. 13 to $k_{j}=0$ for $j \neq l$ is often performed, only taking into account the vibronic structure of the mode excited by the IR beam in the ground state. ${ }^{20,22,69}$ Even if this simplification makes life much easier, it cannot be considered as valid. As a matter of fact, the visible and SFG photons may excite all the symmetry-allowed transitions in the vibronic structure, characterized by their nonvanishing displacements (modes $j$ ). Among these, mode $l$ plays a specific role, but it cannot be considered alone in the vibronic structure. This illustrates the great advantage of embedding the SOS calculations into the overlap spectral function as is done in the TT and TDM formalisms.

\section{DR-SFG AND DR-DFG WITH MODE DISTORTION}

The previous expressions must be completed when quadratic electron-vibration coupling is taken into account. We first extend the results above, as was done for RRS, ${ }^{54}$ to mode distortions (superscripts DIS) without Duschinsky rotation, i.e. quadratic coupling leading to a change in the harmonic potentials and to frequency shifts in the excited state $\left(\omega_{j}^{e} \neq \omega_{j}^{g}\right)$. Starting from Eq. A5, the calculation of the SOS part (i.e. the sum over $\{u\}$ ) is detailed in Appendix B. It is shown that the hyperpolarizability for DR-SFG and DR-DFG can still be recast under the form:

$$
\beta_{i j k}^{S F G}=1 / \hbar^{2} \sum_{l}^{I R-a c t i v e} \frac{A_{l}^{i j k} D_{l}^{D I S}\left(\omega_{S F G}\right)}{\omega_{I R}-\omega_{l}^{g}+i \Gamma_{l}^{g}}
$$

and

$$
\beta_{i j k}^{D F G}=1 / \hbar^{2} \sum_{l}^{I R-a c t i v e} \frac{A_{l}^{i j k} D_{l}^{D I S}\left(\omega_{D F G}+\omega_{l}^{g}\right)}{\omega_{I R}-\omega_{l}^{g}-i \Gamma_{l}^{g}}
$$

where the excitation spectrum including full mode distortion (DIS) becomes

$$
D_{l}^{D I S}(\omega)=\frac{1}{i} \int_{0}^{\infty} d t e^{i t\left(\omega-\omega_{e g}^{0}+i \Gamma_{e g}\right)} \tilde{g}_{l}^{D I S}(t) \prod_{\substack{j=1 \\ j \neq l}}^{N} g_{j}^{D I S}(t)
$$


with

$$
g_{j}^{D I S}(t)=\frac{2 \sqrt{\lambda_{j}}}{\sqrt{\left(1+\lambda_{j}\right)^{2}-w_{j}^{2}\left(1-\lambda_{j}\right)^{2}}} e^{2 S_{j} h_{j}^{D I S}(t)}
$$

and

$$
\tilde{g}_{l}^{D I S}(t)=\Delta_{l} h_{l}^{D I S}(t) g_{l}^{D I S}(t)
$$

Displacement $\Delta_{l}$ is still defined by $Q_{l}^{e}=Q_{l}^{g}+\Delta_{l}$. The following notations are introduced in Appendix B: $w_{j}=e^{-i \omega_{j}^{e} t}$ accounts for the excitation of one quantum in the $j$-th vibration mode of the excited state; $\lambda_{j}=\omega_{j}^{e} / \omega_{j}^{g}$ describes the intensity of the distortion of the $j$-th vibration mode in the electronic excited state; function $h_{j}(t)$ is now defined as:

$$
h_{j}^{D I S}(t)=-1+\frac{1+w_{j}}{1+w_{j}+\lambda_{j}\left(1-w_{j}\right)}
$$

In the Supplementary Material, we show that Equations 14 to 19, obtained by a direct calculation, are identical as expected to the results derived by the transform technique. ${ }^{39}$

Contrary to the linear coupling case, the excitation spectrum does not appear at this stage as a sum and product of Lorentzian functions because of the new form of $h_{j}(t)$. However, such a recasting under a friendlier Lorentzian form becomes possible for low distortions (i.e. small quadratic electron-vibration coupling) by introduction of $\Phi^{F S}(\omega)$, a modified version of the $\Phi^{L C}(\omega)$ function including frequency shifts (FS). $\Phi^{F S}$ is formally calculated using the same equations 10 or 12 as $\Phi^{L C}$, but now involving the shifted values of vibronic frequencies $\omega_{j}^{e}$. When parameter $\lambda_{j}$ is close to 1 (typical values range between 0.9 and 1.1), performing a Taylor expansion in $\left(1-\lambda_{j}\right)$ and keeping only the first order terms, we have:

$$
\begin{gathered}
h_{j}^{D I S}(t) \approx-\frac{1}{2}\left(1-w_{j}\right)+\frac{1}{4}\left(1-\lambda_{j}\right)\left(1-w_{j}^{2}\right) \\
g_{j}^{D I S}(t) \approx e^{-S_{j}\left(1-w_{j}\right)+\frac{1}{2} S_{j}\left(1-\lambda_{j}\right)\left(1-w_{j}^{2}\right)}=g_{j}^{F S}(t)\left[1+\frac{1}{2} S_{j}\left(1-\lambda_{j}\right)\left(1-e^{-2 i \omega_{j}^{e} t}\right)\right]
\end{gathered}
$$

and

$$
\prod_{\substack{j=1 \\ j \neq l}}^{N} g_{j}^{D I S}(t) \approx\left(\prod_{\substack{j=1 \\ j \neq l}}^{N} g_{j}^{F S}(t)\right)\left[1+\frac{1}{2} \sum_{\substack{j=1 \\ j \neq l}}^{N} S_{j}\left(1-\lambda_{j}\right)\left(1-e^{-2 i \omega_{j}^{e} t}\right)\right]
$$

where $g_{j}^{F S}$ is formally identical to $g_{j}^{L C}$ (Eq. 5) but now accounts for $\omega_{j}^{e} \neq \omega_{j}^{g}$. In the same way,

$$
\tilde{g}_{l}^{D I S}(t) \approx-\frac{\Delta_{l}}{2}\left(\left(1-w_{l}\right)-\frac{1}{2}\left(1-\lambda_{l}\right)\left(1-w_{l}^{2}\right)\right) g_{l}^{F S}(t)\left[1+\frac{1}{2} S_{l}\left(1-\lambda_{l}\right)\left(1-w_{l}^{2}\right)\right]
$$




$$
\tilde{g}_{l}^{D I S}(t) \approx-\frac{\Delta_{l}}{2} g_{l}^{F S}(t)\left(1-e^{-i \omega_{l}^{e} t}\right)\left[1-\frac{1}{2}\left(1-\lambda_{l}\right)\left(1+e^{-i \omega_{l}^{e} t}\right)+\frac{1}{2} S_{l}\left(1-\lambda_{l}\right)\left(1-e^{-2 i \omega_{l}^{e} t}\right)\right]
$$

Thus, still at first order in $\left(1-\lambda_{j}\right)$, the integrand of the excitation spectrum becomes

$$
\begin{aligned}
\tilde{g}_{l}^{D I S}(t) \prod_{\substack{j=1 \\
j \neq l}}^{N} g_{j}^{D I S}(t) \approx-\frac{\Delta_{l}}{2}\left(\prod_{j=1}^{N} g_{j}^{F S}(t)\right)\left(1-e^{-i \omega_{l}^{e} t}\right) & {\left[\frac{1}{2}\left(1+\lambda_{l}\right)-\frac{1}{2}\left(1-\lambda_{l}\right) e^{-i \omega_{l}^{e} t}\right.} \\
+ & \left.\frac{1}{2} \sum_{j=1}^{N} S_{j}\left(1-\lambda_{j}\right)\left(1-e^{-2 i \omega_{j}^{e} t}\right)\right]
\end{aligned}
$$

and the excitation spectrum itself therefore simplifies into

$$
\begin{gathered}
D_{l}^{D I S}(\omega)=\frac{\Delta_{l}}{4}\left(\left(1+\lambda_{l}\right) \Phi^{F S}(\omega)-2 \Phi^{F S}\left(\omega-\omega_{l}^{e}\right)+\left(1-\lambda_{l}\right) \Phi^{F S}\left(\omega-2 \omega_{l}^{e}\right)\right. \\
\left.+\sum_{j=1}^{N} S_{j}\left(1-\lambda_{j}\right)\left[\Phi^{F S}(\omega)-\Phi^{F S}\left(\omega-\omega_{l}^{e}\right)-\Phi^{F S}\left(\omega-2 \omega_{j}^{e}\right)+\Phi^{F S}\left(\omega-\omega_{l}^{e}-2 \omega_{j}^{e}\right)\right]\right)
\end{gathered}
$$

Going back to Eq. 14 and 15, it is possible to express the hyperpolarizabilities in SFG and DFG as sums and products of energy denominators with few unknown parameters. The only prerequisite here is the calculation of the overlap spectral function $\Phi^{F S}(\omega)$. To investigate the effects of distortion, we concentrate on the fundamental processes and neglect the HuangRhys contribution. Removing all the higher order $S_{j}$ terms, we have, in the linear coupling scheme:

$$
D_{l}^{L C, \text { fundamental }}(\omega)=-\frac{\Delta_{l}}{2}\left[\frac{1}{\omega-\omega_{e g}^{0}+i \Gamma_{e g}}-\frac{1}{\omega-\omega_{e g}^{0}-\omega_{l}^{e}+i \Gamma_{e g}}\right]
$$

and in the quadratic distorted scheme:

$$
\begin{aligned}
D_{l}^{D I S, f \text { fundamental }}(\omega)= & -\frac{\Delta_{l}}{2}\left(\frac{1+\lambda_{l}}{2}\right)\left[\frac{1}{\omega-\omega_{e g}^{0}+i \Gamma_{e g}}-\frac{1}{\omega-\omega_{e g}^{0}-\omega_{l}^{e}+i \Gamma_{e g}}\right] \\
& -\frac{\Delta_{l}}{2}\left(\frac{1-\lambda_{l}}{2}\right)\left[\frac{1}{\omega-\omega_{e g}^{0}-\omega_{l}^{e}+i \Gamma_{e g}}-\frac{1}{\omega-\omega_{e g}^{0}-2 \omega_{l}^{e}+i \Gamma_{e g}}\right]
\end{aligned}
$$

We first see in Eq. 28, or in the same way in Eq. 26, the effect of the distortion for mode $l$, which allows the excitation of the first overtone for this mode, thus generating a new resonance peak in the excitation spectrum. The overall amplitude of the $\left(\mathrm{g}, \nu_{l}=1\right) \rightarrow\left(\mathrm{e}, \nu_{l}=1\right)$ transition remains constant, whereas the growth of the $\left(\mathrm{g}, \nu_{l}=1\right) \rightarrow\left(\mathrm{e}, \nu_{l}=2\right)$ transition as a function of increasing $\left(1-\lambda_{l}\right)$ happens to the prejudice of the $\left(\mathrm{g}, \nu_{l}=1\right) \rightarrow\left(\mathrm{e}, \nu_{l}=0\right)$ process. The same distortion-allowed excitation of state $\left(\mathrm{e}, \nu_{j}=2\right)$ also happens for all modes $j \neq l$, 
modulated by the coupling constants $S_{j}$. This is well illustrated for a system with only one IR-active mode, for example a diatomic molecule. The excitation spectrum takes the value:

$$
\begin{array}{r}
D_{1}^{D I S}(\omega)=\frac{\Delta_{1}}{4}\left[\left(1+\lambda_{1}+\left(1-\lambda_{1}\right) S_{1}\right) \Phi^{F S}(\omega)-\left(2+\left(1-\lambda_{1}\right) S_{1}\right) \Phi^{F S}\left(\omega-\omega_{1}^{e}\right)\right. \\
\left.+\left(1-S_{1}\right)\left(1-\lambda_{1}\right) \Phi^{F S}\left(\omega-2 \omega_{1}^{e}\right)+S_{1}\left(1-\lambda_{1}\right) \Phi^{F S}\left(\omega-3 \omega_{1}^{e}\right)\right]
\end{array}
$$

In the development of $D_{l}^{D I S}(\omega)$, for a given nonvanishing order $k_{l}$ of the Huang-Rhys factor $S_{l}$, there are in total four different vibronic transitions which enter the doubly resonant process, with energies ranging from $\left(k_{l}-1\right) \omega_{l}^{e}$ to $\left(k_{l}+2\right) \omega_{l}^{e}$ above $\omega_{e g}^{0}$.

We can now analyze the various terms and processes involved in the electronic excitation spectrum. When neglecting mode distortion, the fundamental process is resonant with levels $\left(\mathrm{e}, \nu_{l}=0\right)$ and $\left(\mathrm{e}, \nu_{l}=1\right)$ of the excited state (Eq. 27). In the time correlator formalism, this follows from the $h_{l}(t)$ function in $\tilde{g}_{l}(t)$, or from functions $\Psi_{1}$ and $\Psi_{2}$ as defined in the Supplementary Material. ${ }^{39}$ At higher orders of vibronic coupling, overtones and combination states may be reached, weighted by coupling constants $S_{j}$, once per vibrational quantum. These processes are described by $S_{j} h_{j}(t)$ in $g_{j}(t)$ in Eq. 4 and 5 , and by $F_{c}$ and $F_{d}$ terms in the Supplementary Material. In other words, $S_{j}$ triggers the $\left(\mathrm{e}, \nu_{j} \rightarrow \nu_{j+1}\right)$ process accounted for by $h_{j}(t)$.

Vibronic mode distortion enables to reach new states of increasing energy for a given order of $S_{j}$ by adding new terms to $h_{j}(t)$. Equations 20 and 21 show that the development of $h_{j}(t)$ as a function of increasing powers of $\left(1-\lambda_{j}\right)$ allows to reach successive overtones of increasing order of mode $j$. Therefore, for low distortion, additional resonant processes involve the second harmonics of mode $l$ in the fundamental process (Eq. 28), and that of modes $j$ at first order of $S_{j}$ (instead of second order) in the correcting terms of Eq. 26 and 29. By extension, the full development of $h_{j}(t)$ functions contains the whole excitation of vibronic state $j$ and its harmonics in a single jump during the DR-FG process, which probabilities are weighted by increasing powers of $\left(1-\lambda_{j}\right)$ and therefore rapidly fade out.

\section{DR-SFG AND DR-DFG WITH MODE MIXING AND MODE DISTORTION}

We now introduce mode mixing into the previous description. The general relationship between normal mode coordinates becomes $\mathbf{Q}_{\mathbf{e}}=\mathbf{R Q}_{\mathrm{g}}+\boldsymbol{\Delta}^{39,57}$ where normal coordinates 
for ground and excited states are grouped in N-dimension vectors $\mathbf{Q}, \mathbf{R}$ is the Duschinsky rotation matrix and $\Delta$ is the vector of the equilibrium position shifts $\Delta_{j}$. Although a direct calculation of Franck-Condon integrals through a SOS procedure seems possible and computable, ${ }^{37,40,41,51-53,83}$ we follow here the results of Vallet et al. ${ }^{39}$, applying the transform technique to SFG and DFG. We first limit the analysis (see Appendix C for details) to a multimode system in which two modes (numbered 1 and 2) are involved in a Duschinsky rotation (angle $\theta$ ), under the hypothesis of small rotation amplitudes because the general case for an arbitrary angle does not allow a clear separation of resonant terms. Extension to a more general system involving pairs of mixed vibration modes is discussed below. In the case of mode mixing, there is no direct proportionality between quantities related to vibrational coordinates in the lower and upper states, and several useful quantities (i.e. normal mode displacements in the ground state basis $\Delta_{j}, L_{j}^{\prime}, L_{j}^{\prime \prime}$, and in the excited state basis $L_{j}, \xi_{j}$ ) have been defined throughout the literature. ${ }^{39,63,89,90}$ As a consequence, one must choose among these the appropriate parameters to quantify the displacements along the vibration coordinates. Following the derivation in the Appendix C and Supplementary Material, and the discussion on that topic in the literature, ${ }^{56}$ the most convenient parameters are $\Delta_{j}$, the overall equilibrium displacements under electronic excitation for the general quadratic model. ${ }^{39}$

The general forms for the SFG and DFG hyperpolarizabilities are given in the Appendix C for arbitrary mode distortion in integral form (Eq. C3). For small mode distortion, keeping only the first order terms in $\left(1-\lambda_{j}\right)$ and $\theta$, it is again possible to recover a more conventional form with products of Lorentzian functions. Under this hypothesis, as demonstrated in Appendix $\mathrm{C}$, the hyperpolarizabilities take the form

$$
\beta_{i j k}^{S F G}=\frac{1}{\hbar^{2}}\left(\sum_{l}^{I R-a c t i v e} \frac{A_{l}^{i j k} D_{l}^{M I X}\left(\omega_{S F G}\right)}{\omega_{I R}-\omega_{l}^{g}+i \Gamma_{l}^{g}}+\frac{A_{1}^{i j k} D_{1}^{C T}\left(\omega_{S F G}\right)}{\omega_{I R}-\omega_{1}^{g}+i \Gamma_{1}^{g}}+\frac{A_{2}^{i j k} D_{2}^{C T}\left(\omega_{S F G}\right)}{\omega_{I R}-\omega_{2}^{g}+i \Gamma_{2}^{g}}\right)
$$

and

$$
\begin{aligned}
\beta_{i j k}^{D F G}=\frac{1}{\hbar^{2}} & \left(\sum_{l}^{I R} \frac{A_{l}^{i j k} D_{l}^{M I X}\left(\omega_{D F G}+\omega_{l}^{g}\right)}{\omega_{I R}-\omega_{l}^{g}-i \Gamma_{l}^{g}}\right. \\
& \left.+\frac{A_{1}^{i j k} D_{1}^{C T}\left(\omega_{D F G}+\omega_{1}^{g}\right)}{\omega_{I R}-\omega_{1}^{g}-i \Gamma_{1}^{g}}+\frac{A_{2}^{i j k} D_{2}^{C T}\left(\omega_{D F G}+\omega_{2}^{g}\right)}{\omega_{I R}-\omega_{2}^{g}-i \Gamma_{2}^{g}}\right)
\end{aligned}
$$


with $D_{l}^{M I X}(\omega)=D_{l}^{D I S}(\omega)+D_{l}^{\theta}(\omega), D_{l}^{D I S}(\omega)$ refers to Eq. 26,

$$
\begin{aligned}
& D_{l}^{\theta}(\omega)=-\frac{\Delta_{l} \Delta_{1} \Delta_{2} \theta\left(\omega_{1}^{g}-\omega_{2}^{g}\right)}{4 \hbar}\left[\Phi^{F S}(\omega)-\Phi^{F S}\left(\omega-\omega_{l}^{e}\right)\right. \\
& -\Phi^{F S}\left(\omega-\omega_{1}^{e}\right)+\Phi^{F S}\left(\omega-\omega_{l}^{e}-\omega_{1}^{e}\right) \\
& -\Phi^{F S}\left(\omega-\omega_{2}^{e}\right)+\Phi^{F S}\left(\omega-\omega_{l}^{e}-\omega_{2}^{e}\right) \\
& \left.+\Phi^{F S}\left(\omega-\omega_{1}^{e}-\omega_{2}^{e}\right)-\Phi^{F S}\left(\omega-\omega_{l}^{e}-\omega_{1}^{e}-\omega_{2}^{e}\right)\right], \\
& D_{1}^{C T}(\omega)=+\frac{\Delta_{2} \theta}{4 \omega_{1}^{g}}\left\{\left(\omega_{1}^{g}+\omega_{2}^{g}\right)\left[\Phi^{F S}(\omega)-\Phi^{F S}\left(\omega-\omega_{2}^{e}\right)\right]\right. \\
& \left.-\left(\omega_{1}^{g}-\omega_{2}^{g}\right)\left[\Phi^{F S}\left(\omega-\omega_{1}^{e}\right)-\Phi^{F S}\left(\omega-\omega_{1}^{e}-\omega_{2}^{e}\right)\right]\right\},
\end{aligned}
$$

and

$$
\begin{aligned}
D_{2}^{C T}(\omega)=-\frac{\Delta_{1} \theta}{4 \omega_{2}^{g}}\left\{\left(\omega_{1}^{g}+\omega_{2}^{g}\right)\left[\Phi^{F S}(\omega)-\Phi^{F S}\left(\omega-\omega_{1}^{e}\right)\right]\right. & \\
& \left.-\left(\omega_{2}^{g}-\omega_{1}^{g}\right)\left[\Phi^{F S}\left(\omega-\omega_{2}^{e}\right)-\Phi^{F S}\left(\omega-\omega_{1}^{e}-\omega_{2}^{e}\right)\right]\right\} .
\end{aligned}
$$

The numerators appear as simple linear combinations of function $\Phi^{F S}(\omega)$ evaluated at several wavenumbers. In particular, $D_{l}^{M I X}$ is the sum of $D_{l}^{D I S}$ and a term $D_{l}^{\theta}$, proportional to $\theta$ and belonging to the higher order contributions, whereas $D^{C T}$ do not depend on distortion parameters. The effects of mode distortion on the SFG amplitudes, beyond frequency shifts, are therefore separated from mode mixing contributions. We note that the $\theta$-dependent corrective term for mode 1 is proportional to $\Delta_{2}$, and vice versa. When $\Delta_{1}$ and $\Delta_{2}$ significantly differ in magnitude, we may expect $D_{2}^{C T}$ to become bigger than the main $D_{1}^{M I X}$ contributions (or the other way round) and have a major impact on the activity of mode 1.

In this compact form, a single calculation of the overlap spectral function $\Phi^{F S}$ leads to a fast access to the SFG and DFG hyperpolarizabilities including mode mixing and mode distortion, which appear here as two distinct perturbations of the linear coupling scheme.

The significances of the additional terms in Eq. 30 and 31 become clear when neglecting the Huang-Rhys contribution (together with the terms driven by the $\Delta_{1} \Delta_{2}$ product) and keeping to the fundamental process, leading to:

$$
D_{l}^{M I X, \text { fundamental }}(\omega)=D_{l}^{D I S}(\omega)
$$

This proves that, for a generic mode $l \neq 1,2$, mode mixing only impacts the higher order vibronic transitions but has no effect on the fundamental process. On the contrary, for mode 
1 (and symmetrically for mode 2):

$$
\begin{aligned}
& D_{1}^{C T, \text { fundamental }}(\omega)=-\frac{\Delta_{2} \theta}{4 \omega_{1}^{g}} e^{-S} \sum_{p=0}^{p=1}(-1)^{p}\left(\frac{\omega_{1}^{g}+\omega_{2}^{g}}{\omega-\omega_{0}^{e g}-p \omega_{2}^{e}+i \Gamma_{e g}}\right. \\
& \left.-\frac{\omega_{1}^{g}-\omega_{2}^{g}}{\omega-\omega_{0}^{e g}-p \omega_{2}^{e}-\omega_{1}^{e}+i \Gamma_{e g}}\right)
\end{aligned}
$$

Again, mode mixing introduces new allowed states in the fundamental process. As could have been expected, they consist of the direct excitation of mode $\left(\mathrm{e}, \nu_{2}=1\right)$ from $\left(\mathrm{g}, \nu_{1}=1\right)$ and vice versa, which if forbidden when mixing vanishes. It also allows to reach state $\left(\mathrm{e}, \nu_{1}=1, \nu_{2}=1\right)$ at the same order of coupling. This comes from the fact that a vertical jump from $\left(\mathrm{g}, \nu_{1}=1\right)$ reaches a combination state of modes 1 and 2 . The same phenomenon happens at higher order of vibronic coupling for all modes $l$, as evidenced by the correcting term in $D_{l}^{M I X}(\omega)$.

Finally, it is possible to generalize the above calculation to the case where $\mathrm{P}$ vibration modes in the excited state are involved in a small amplitude Duschinsky rotation by pairs, i.e. $(1,2) ;(3,4) ; \ldots ;(\mathrm{P}-1, \mathrm{P})$ with $\mathrm{P} \leq \mathrm{N}$. This is usually a good approximation to real systems. ${ }^{48}$ For the reasons exposed in the Appendix C, this simply results in the addition of the (P-1) corresponding $D^{C T}$ terms in Eq. 30 and 31, and $\mathrm{P} / 2$ in each $D_{l}^{M I X}(\omega)$ for modes 3,4,P-1,P. The SFG hyperpolarizability in this general case is given by

$$
\beta_{i j k}^{S F G}=\frac{1}{\hbar^{2}} \sum_{l}^{I R-a c t i v e} \frac{A_{l}^{i j k}}{\omega_{I R}-\omega_{l}^{g}+i \Gamma_{l}^{g}}\left[D_{l}^{D I S}\left(\omega_{S F G}\right)+D_{l}^{\theta}\left(\omega_{S F G}\right)+D_{l}^{C T}\left(\omega_{S F G}\right)\right]
$$

where $D_{l}^{D I S}(\omega)$ is still given by Eq. $26, D_{l}^{\theta}(\omega)$ now sums up all the contributions from $(\mathrm{i}, \mathrm{j})$ pairs of mixed modes (angle $\theta_{i j}$ ) and becomes

$$
\begin{gathered}
D_{l}^{\theta}(\omega)=-\Delta_{l} \sum_{(i, j)=(1,2)}^{(P-1, P)} \frac{\Delta_{i} \Delta_{j} \theta_{i j}\left(\omega_{i}^{g}-\omega_{j}^{g}\right)}{4 \hbar}\left[\Phi^{F S}(\omega)-\Phi^{F S}\left(\omega-\omega_{l}^{e}\right)\right. \\
-\Phi^{F S}\left(\omega-\omega_{i}^{e}\right)+\Phi^{F S}\left(\omega-\omega_{l}^{e}-\omega_{i}^{e}\right) \\
-\Phi^{F S}\left(\omega-\omega_{j}^{e}\right)+\Phi^{F S}\left(\omega-\omega_{l}^{e}-\omega_{j}^{e}\right) \\
\left.\quad+\Phi^{F S}\left(\omega-\omega_{i}^{e}-\omega_{j}^{e}\right)-\Phi^{F S}\left(\omega-\omega_{l}^{e}-\omega_{i}^{e}-\omega_{j}^{e}\right)\right],
\end{gathered}
$$

while $D_{l}^{C T}(\omega)$ vanishes for modes $l$ not involved in mode mixing and becomes

$$
\begin{aligned}
D_{i}^{C T}(\omega)=+\frac{\Delta_{j} \theta_{i j}}{4 \omega_{i}^{g}}\left\{\left(\omega_{i}^{g}+\omega_{j}^{g}\right)\right. & {\left[\Phi^{F S}(\omega)-\Phi^{F S}\left(\omega-\omega_{j}^{e}\right)\right] } \\
& \left.-\left(\omega_{i}^{g}-\omega_{j}^{g}\right)\left[\Phi^{F S}\left(\omega-\omega_{i}^{e}\right)-\Phi^{F S}\left(\omega-\omega_{i}^{e}-\omega_{j}^{e}\right)\right]\right\},
\end{aligned}
$$


and

$$
\begin{aligned}
D_{j}^{C T}(\omega)=-\frac{\Delta_{i} \theta_{i j}}{4 \omega_{j}^{g}}\left\{\left(\omega_{i}^{g}+\omega_{j}^{g}\right)[\right. & \left.\Phi^{F S}(\omega)-\Phi^{F S}\left(\omega-\omega_{i}^{e}\right)\right] \\
& \left.-\left(\omega_{j}^{g}-\omega_{i}^{g}\right)\left[\Phi^{F S}\left(\omega-\omega_{j}^{e}\right)-\Phi^{F S}\left(\omega-\omega_{i}^{e}-\omega_{j}^{e}\right)\right]\right\}
\end{aligned}
$$

for the first and second mode of any $(\mathrm{i}, \mathrm{j})$ pair, respectively.

\section{CONCLUSION}

In singly IR-resonant FG spectroscopy, experimental spectra are recorded in a rather narrow infrared range, and the number of vibration modes involved is limited to a few. Careful data fitting and polarization analysis remains accessible, as it only depends on a little number of parameters per mode, even if interference patterns between neighboring modes, and with a nonresonant contribution, may become challenging even for specialists. In a second step, it is possible to calculate, for example by DFT methods, the IR and Raman activities of the vibration modes, helping in the analysis of molecular orientations, intermolecular couplings or the influence of the substrate. ${ }^{91}$ Doubly resonant molecular processes in SFG and DFG spectroscopies appear much more complex, even in the linear electron-vibration coupling frame. As in RRS, they bring in optical resonances with the full molecular vibronic structure. As far as first principle calculations are involved, one must increase in that case both the number of calculated vibration modes and the level of ab initio methods (e.g. TD-DFT, CCSD), in order to determine the parameters of the electronic transition and the vibrational displacements along the normal mode coordinates. ${ }^{77,92,93}$

These $a b$ initio calculations of DR-FG spectra do not take into account mode distortions and mode mixing. Firstly, the theoretical grounds and equations building up the hyperpolarizabilities in that case were missing up to now, and, secondly, this raises additional complexity for first principle calculations. As a matter of fact, it becomes necessary to calculate, in addition to the previous quantities, the full molecular vibronic structure in order to determine the amplitudes of mode distortions, and which modes are mixed in the excited state, in other words $\lambda_{j}$ parameters and the Duschinsky matrix. With the constant evo-

lution of available methods and computational power, these parameters become accessible, opening the door to a full account of the structural complexity of vibronic transitions. 
In this paper, we have investigated the effects of quadratic electron-vibration couplings, responsible for mode distortions and mode mixing, on the simulated DR-FG spectra. For small coupling amplitudes, both phenomena may be included in the excitation spectrum of the IR-resonant vibration modes through a modification of the linear coupling equations. We have shown that, for small distortion amplitudes, we may describe the consequences of mode distortions by their induced shifts in vibration frequencies while the other effects (e.g. excitation of overtones and combination vibronic states) have a limited impact on the excitation spectra. On the contrary, mode mixing, even for low mixing angles, deeply influences the excitation spectra of the mixed modes, leading to a modification of the balance between their DR-FG amplitudes as a function of the mixing parameter. We believe that it is possible to account for these effects in data analysis of actual DR-FG spectra coupled to extensive ab initio calculations of the vibronic structure.

\section{SUPPLEMENTARY MATERIAL}

See Supplementary Material for details on the calculation of Franck-Condon overlap integrals, on the equivalence of sum-over-states and transform technique formalisms, and on the implementation of mode mixing.

\section{ACKNOWLEDGMENTS}

We thank Dr. Abderrahmane Tadjeddine and Dr. Thomas Noblet for their fruitful comments on this work and their suggestions of improvements on this manuscript.

\section{DATA AVAILABILITY}

Data sharing is not applicable to this article as no new data were created or analyzed in this study.

\section{Appendix A: Review of the basic equations for DR-SFG and DR-DFG}

We consider the IR-visible SFG and DFG hyperpolarizabilities in the Born-Oppenheimer, harmonic vibrations and Condon approximations for vanishing temperature (molecule 
in the vibrationless ground state $g 0$ ), with a single molecular excited electronic state (e). Retaining only the doubly resonant hyperpolarizability contributions for component $\beta_{i j k}\left(\omega_{F G} ; \omega_{v i s}, \omega_{I R}\right)$, where $(\mathrm{i}, \mathrm{j}, \mathrm{k})$ stand for cartesian components in the molecular frame:

$$
\begin{aligned}
& \beta_{i j k}^{S F G}=1 / \hbar^{2} \sum_{m,\{v\}} \frac{\mu_{g v \leftarrow g 0}^{k}}{\omega_{I R}-\omega_{g v g 0}+i \Gamma_{g v g 0}} \frac{\mu_{g 0 \leftarrow m}^{i} \mu_{m \leftarrow g v}^{j}}{\omega_{S F G}-\omega_{m g 0}+i \Gamma_{m g 0}} \\
& \beta_{i j k}^{D F G}=1 / \hbar^{2} \sum_{m,\{v\}} \frac{\mu_{g 0 \leftarrow g v}^{k}}{\omega_{I R}-\omega_{g v g 0}-i \Gamma_{g v g 0}} \frac{\mu_{m \leftarrow g 0}^{j} \mu_{g v \leftarrow m}^{i}}{\omega_{D F G}-\omega_{m g v}+i \Gamma_{m g v}}
\end{aligned}
$$

where $\omega_{a b}=\left(E_{a}-E_{b}\right) / \hbar$ is the energy difference (in wavenumber unit) between states $a$ and $b ; \Gamma_{a b}>0$ the damping rate of the $a \leftarrow b$ transition; $\mu_{a \leftarrow b}^{i / j / k}=\left\langle a\left|\mu^{i / j / k}\right| b\right\rangle$ the dipolar transition moment between states $\mathrm{b}$ and a, projected along i,j or k; $g$ the ground electronic state; $\{\nu\}$ spans the whole vibrational structure of state $g ; m$ an intermediate state. At $\mathrm{T}=0$ $\mathrm{K}$, summation over $g$ disappears and recasting of dummy indices is therefore possible ${ }^{17}$. The second term of the product involves the vibronic structure of the molecule.

For small vibrational amplitudes, the IR dipolar transition moment reduces to

$$
\mu_{g v \leftarrow g 0}^{k}=\left\langle g v\left|\mu^{k}\right| g 0\right\rangle
$$

with $\mu^{k}=\mu_{0}^{k}+\sum_{l=1}^{N}\left(\frac{\partial \mu^{k}}{\partial Q_{l}^{g}}\right)_{Q_{l}^{g}=0} Q_{l}^{g}+\ldots$ where $\mathrm{N}$ is the number of vibration modes and $Q_{l}^{g}$ the vibration coordinate for normal mode $l$ in the ground state. The first term vanishes because of the orthogonality of vibrational wavefunctions, and

$$
\mu_{g v \leftarrow g 0}^{k} \approx \sum_{l=1}^{I R-a c t i v e}\left(\frac{\partial \mu^{k}}{\partial Q_{l}^{g}}\right)_{Q_{l}^{g}=0}\left\langle g v\left|Q_{l}^{g}\right| g 0\right\rangle
$$

In the harmonic approximation, sum over $\{v\}$ reduces to one term corresponding to the creation of one vibration quantum for mode $l$. The sum over $m$ now runs over the entire vibrational structure $\{u\}$ of the upper electronic state $e$.

$$
\beta_{i j k}^{S F G}=1 / \hbar^{2} \sum_{l}^{I R-a c t i v e} \frac{\left(\frac{\partial \mu^{k}}{\partial Q_{l}^{g}}\right)_{Q_{l}^{g}=0} \sqrt{\frac{\hbar}{2 \omega_{l}^{g}}}}{\omega_{I R}-\omega_{l}^{g}+i \Gamma_{l}^{g}} \sum_{\{u\}} \frac{\mu_{g 0 \leftarrow e u}^{i} \mu_{e u \leftarrow g l}^{j}}{\omega_{S F G}-\omega_{e u g}+i \Gamma_{e g}}
$$

In the same way,

$$
\beta_{i j k}^{D F G}=1 / \hbar^{2} \sum_{l}^{I R-a c t i v e} \frac{\left(\frac{\partial \mu^{k}}{\partial Q_{l}^{g}}\right)_{Q_{l}^{g}=0} \sqrt{\frac{\hbar}{2 \omega_{l}^{g}}}}{\omega_{I R}-\omega_{l}^{g}-i \Gamma_{l}^{g}} \sum_{\{u\}} \frac{\mu_{g l \leftarrow e u}^{i} \mu_{e u \leftarrow g 0}^{j}}{\omega_{D F G}-\omega_{e u g l}+i \Gamma_{e g}}
$$




\section{Appendix B: DR-FG excitation spectrum including mode distortions}

Starting from Eq. A5 and separating the electronic and vibrational parts of the wave

function in the Born-Oppenheimer approximation, we note $|g l\rangle=\left|\psi_{g}^{e l e c} \psi_{l}^{v i b, g}\right\rangle$ and $\mu_{e u \leftarrow g l}^{j}=$ $\left\langle\psi_{e}^{\text {elec }} \psi_{u}^{v i b, e}\left|\mu_{e \leftarrow g}^{j}\right| \psi_{g}^{\text {elec }} \psi_{l}^{v i b, g}\right\rangle$. A distinction is now made between the vibration coordinates $Q_{l}^{g}$ and $Q_{l}^{e}$, which relate by $Q_{l}^{e}=Q_{l}^{g}+\Delta_{l}$. In the harmonic approximation,

$$
\left|\psi_{l}^{v i b, g}\right\rangle=\left|\psi_{l, 1}^{\text {harm }}\left(Q_{l}^{g}\right)\right\rangle \prod_{\substack{j=1 \\ j \neq l}}^{N}\left|\psi_{j, 0}^{\text {harm }}\left(Q_{j}^{g}\right)\right\rangle \text { and }\left|\psi_{\mathrm{u}}^{\mathrm{vib}, \mathrm{e}}\right\rangle=\sum_{\mathrm{u}_{\mathrm{j}}} \prod_{\mathrm{j}=1}^{\mathrm{N}}\left|\psi_{\mathrm{j}, \mathrm{u}_{\mathrm{j}}}^{\mathrm{harm}}\left(\mathrm{Q}_{\mathrm{j}}^{\mathrm{e}}\right)\right\rangle
$$

where $j$ spans the vibration modes and $u_{j}$ is the quantum number for each mode, with

$$
\psi_{j, u_{j}}^{\text {harm }}\left(Q_{j}^{e, g}\right)=N_{u_{j}} H_{u_{j}}\left(\sqrt{\frac{\omega_{j}^{e, g}}{\hbar}} Q_{j}^{e, g}\right) e^{-\frac{\omega_{j}^{e}}{2 \hbar}\left(Q_{j}^{e, g}\right)^{2}}
$$

$H_{u_{j}}$ is the $u_{j}$-th Hermite polynomial and $N_{u_{j}}$ the normalisation factor equal to

$$
\frac{1}{\sqrt{2^{u_{j}} u_{j} !}}\left(\frac{\omega_{j}^{e, g}}{\hbar \pi}\right)^{1 / 4}
$$

Using the Condon approximation, we have for example

$$
\mu_{e u \leftarrow g l}^{j}=\mu_{e \leftarrow g}^{0, j}\left\langle\psi_{u}^{v i b, e} \mid \psi_{l}^{v i b, g}\right\rangle
$$

and the electronic part of the transitions are embedded in the constant transition moments.

$$
\begin{gathered}
\mu_{g 0 \leftarrow e u}^{i} \mu_{e u \leftarrow g l}^{j}=\mu_{g \leftarrow e}^{0, i} \mu_{e \leftarrow g}^{0, j}\left\langle\psi_{0}^{v i b, g} \mid \psi_{u}^{v i b, e}\right\rangle\left\langle\psi_{u}^{v i b, e} \mid \psi_{l}^{v i b, g}\right\rangle \\
\beta_{i j k}^{S F G}=\frac{1}{\hbar^{2}} \sum_{l}^{I R-a c t i v e} \frac{A_{l}^{i j k} \sqrt{\frac{\hbar}{2 \omega_{l}^{g}}}}{\omega_{I R}-\omega_{l}^{g}+i \Gamma_{l}^{g}} \sum_{\{u\}} \frac{\left\langle\psi_{0}^{v i b, g} \mid \psi_{u}^{v i b, e}\right\rangle\left\langle\psi_{u}^{v i b, e} \mid \psi_{l}^{v i b, g}\right\rangle}{\omega_{S F G}-\omega_{e u g 0}+i \Gamma_{e g}}
\end{gathered}
$$

with $A_{l}^{i j k}=\mu_{g \leftarrow e}^{0, i} \mu_{e \leftarrow g}^{0, j}\left(\frac{\partial \mu^{k}}{\partial Q_{l}^{g}}\right)_{Q_{l}^{g}=0}$ and, for a given set of $\left\{u_{j}\right\}$

$$
\begin{aligned}
\left\langle\psi_{0}^{v i b, g} \mid \psi_{u}^{v i b, e}\right\rangle\left\langle\psi_{u}^{v i b, e} \mid \psi_{l}^{v i b, g}\right\rangle=\left\langle\psi_{l, 0}^{\text {harm }}\left(Q_{l}^{g}\right) \mid \psi_{l, u_{l}}^{\text {harm }}\left(Q_{l}^{e}\right)\right\rangle\left\langle\psi_{l, u_{l}}^{\text {harm }}\left(Q_{l}^{e}\right) \mid \psi_{l, 1}^{\text {harm }}\left(Q_{l}^{g}\right)\right\rangle & \\
& \times \prod_{\substack{j=1 \\
j \neq l}}^{N}\left|\left\langle\psi_{j, 0}^{\text {harm }}\left(Q_{j}^{g}\right) \mid \psi_{j, u_{j}}^{\text {harm }}\left(Q_{j}^{e}\right)\right\rangle\right|^{2}
\end{aligned}
$$

In a general way, we have $\omega_{e u g 0}=\omega_{e g}^{0}+\sum_{j, u_{j}} u_{j} \omega_{j}^{e}$ where $\omega_{e g}^{0}$ is the zero-vibration transition frequency as defined in the text. 
To perform the summation over the $\{u\}$ states, we use the equality $\frac{1}{x}=\frac{1}{i} \int_{0}^{\infty} e^{i t x} d t$, valid when $\mathrm{x}$ has a positive imaginary part, and rewrite

$$
\beta_{i j k}^{S F G}=\frac{1}{i \hbar^{2}} \sum_{l}^{I R} \frac{A_{l}^{i j k} \sqrt{\frac{\hbar}{2 \omega_{l}^{g}}}}{\omega_{I R}-\omega_{l}^{g}+i \Gamma_{l}^{g}} \int_{0}^{\infty} d t \sum_{\{u\}}\left\langle\psi_{0}^{v i b, g} \mid \psi_{u}^{v i b, e}\right\rangle\left\langle\psi_{u}^{v i b, e} \mid \psi_{l}^{v i b, g}\right\rangle e^{i t\left(\omega_{S F G}-\omega_{e g}^{0}-\sum_{j, u_{j}} u_{j} \omega_{j}^{e}+i \Gamma_{e g}\right)}
$$

We have assumed here the uniqueness of the damping constants $\Gamma_{\text {eug } 0} \equiv \Gamma_{\text {eg }}$ among the vibrational levels, as discussed in the text. Replacing the transition probabilities by their values and reordering the terms, we have

$$
\beta_{i j k}^{S F G}=\frac{1}{\hbar^{2}} \sum_{l}^{I R-a c t i v e} \frac{A_{l}^{i j k} D_{l}^{D I S}\left(\omega_{S F G}\right)}{\omega_{I R}-\omega_{l}^{g}+i \Gamma_{l}^{g}}
$$

where

$$
\begin{gathered}
D_{l}^{D I S}(\omega)=\frac{1}{i} \int_{0}^{\infty} d t e^{i t\left(\omega-\omega_{e g}^{0}+i \Gamma_{e g}\right)} \tilde{g}_{l}^{D I S}(t) \prod_{\substack{j=1 \\
j \neq l}}^{N} g_{j}^{D I S}(t) \\
\tilde{g}_{l}^{D I S}(t)=\sqrt{\frac{\hbar}{2 \omega_{l}^{g}} \sum_{u_{l}=0}^{\infty}\left\langle\psi_{l, 0}^{\text {harm }}\left(Q_{l}^{g}\right) \mid \psi_{l, u_{l}}^{\text {harm }}\left(Q_{l}^{e}\right)\right\rangle\left\langle\psi_{l, u_{l}}^{\text {harm }}\left(Q_{l}^{e}\right) \mid \psi_{l, 1}^{\text {harm }}\left(Q_{l}^{g}\right)\right\rangle e^{-i t u_{l} \omega_{l}^{e}}}
\end{gathered}
$$

and

$$
g_{j}^{D I S}(t)=\sum_{u_{j}=0}^{\infty}\left|\left\langle\psi_{j, 0}^{\text {harm }}\left(Q_{j}^{g}\right) \mid \psi_{j, u_{j}}^{\text {harm }}\left(Q_{j}^{e}\right)\right\rangle\right|^{2} e^{-i t u_{j} \omega_{j}^{e}}
$$

Evaluating these functions requires to calculate Franck-Condon overlap integrals, ${ }^{40}$ using $Q_{l}^{e}=Q_{l}^{g}+\Delta_{l}$, as described in the Supplementary Material. We introduce parameter $\lambda_{j}=$ $\omega_{j}^{e} / \omega_{j}^{g},{ }^{54}$ which describes the intensity of the distortion of the $j$-th vibration mode in the electronic excited state. It follows

$$
g_{j}^{D I S}(t)=\frac{2 \sqrt{\lambda_{j}}}{\sqrt{\left(1+\lambda_{j}\right)^{2}-w_{j}^{2}\left(1-\lambda_{j}\right)^{2}}} e^{2 S_{j} h_{j}^{D I S}(t)}
$$

where we introduce the new value of function

$$
h_{j}^{D I S}(t)=-1+\frac{1+w_{j}}{1+w_{j}+\lambda_{j}\left(1-w_{j}\right)}
$$

The calculation of $\tilde{g}_{l}^{D I S}(t)$ follows the same tracks and leads to

$$
\tilde{g}_{l}^{D I S}(t)=\Delta_{l} h_{l}^{D I S}(t) \frac{2 \sqrt{\lambda_{l}}}{\sqrt{\left(1+\lambda_{l}\right)^{2}-w_{l}^{2}\left(1-\lambda_{l}\right)^{2}}} e^{2 S_{l} h_{l}^{D I S}(t)}=\Delta_{l} h_{l}^{D I S}(t) g_{l}^{D I S}(t)
$$


In the DFG case, the calculation is led in the same way:

$$
\beta_{i j k}^{D F G}=\frac{1}{\hbar^{2}} \sum_{l}^{I R-\text { active }} \frac{\left(\frac{\partial \mu^{k}}{\partial Q_{l}^{g}}\right)_{Q_{l}^{g}=0} \sqrt{\frac{\hbar}{2 \omega_{l}^{g}}}}{\omega_{I R}-\omega_{l}^{g}-i \Gamma_{l}^{g}} \sum_{\{u\}} \frac{\mu_{g l \leftarrow e u}^{i} \mu_{e u \leftarrow g 0}^{j}}{\omega_{D F G}-\omega_{e u g l}+i \Gamma_{e g}}
$$

with $\omega_{\text {eugl }}=\omega_{e g}^{0}-\omega_{l}^{g}+\sum_{j, u_{j}} u_{j} \omega_{j}^{e}$

$$
\begin{aligned}
& \beta_{i j k}^{D F G}=\frac{1}{i \hbar^{2}} \sum_{l}^{I R} \frac{A_{l}^{i j k} \sqrt{\frac{\hbar}{2 \omega_{l}^{g}}}}{\omega_{I R}-\omega_{l}^{g}-i \Gamma_{l}^{g}} \int_{0}^{\infty} d t \sum_{\{u\}}\left\langle\psi_{l}^{v, g} \mid \psi_{u}^{v, e}\right\rangle\left\langle\psi_{u}^{v, e} \mid \psi_{0}^{v, g}\right\rangle e^{i t\left(\omega_{D F G}-\omega_{e g}^{0}+\omega_{l}^{g}-\sum_{j, u_{j}} u_{j} \omega_{j}^{e}+i \Gamma_{e g}\right)} \\
& \beta_{i j k}^{D F G}=\frac{1}{i \hbar^{2}} \sum_{l}^{I R-a c t i v e} \frac{A_{l}^{i j k}}{\omega_{I R}-\omega_{l}^{g}-i \Gamma_{l}^{g}} \int_{0}^{\infty} d t e^{i t\left(\omega_{D F G}-\omega_{e g}^{0}+\omega_{l}^{g}+i \Gamma_{e g}\right)} \tilde{g}_{l}^{D I S}(t) \prod_{\substack{j=1 \\
j \neq l}}^{N} g_{j}^{D I S}(t) \\
& \beta_{i j k}^{D F G}=\frac{1}{\hbar^{2}} \sum_{l}^{I R-\text { active }} \frac{A_{l}^{i j k} D_{l}^{D I S}\left(\omega_{D F G}+\omega_{l}^{g}\right)}{\omega_{I R}-\omega_{l}^{g}-i \Gamma_{l}^{g}}
\end{aligned}
$$

\section{Appendix C: DR-FG excitation spectrum including mode mixing}

The explicit calculation including both frequency shifts for all modes $j$ (measured by $\left.\lambda_{j}=\omega_{j}^{e} / \omega_{j}^{g}\right)$ and Duschinsky rotation may be performed explicitly at $\mathrm{T}=0 \mathrm{~K}$ under some conditions. We adapt the formulas of Ref. 39 (to which we refer the reader for the notations) and start with the general expression for the hyperpolarizability:

$$
\beta_{i j k}=-\frac{M_{g e}^{i j k}}{\hbar^{2}} \int_{0}^{\infty} d t\left[\Psi_{1}(t)-\Psi_{2}(t)\right] \exp \left[i \Delta \omega t-\Gamma_{e g} t+F_{d}(t)+F_{c}(t)\right]
$$

The link between this equation and our notations is detailed in the Supplementary Material, as well as the meaning of the various terms.

We first restrict the calculation to the following context:

- only two modes (numbered 1 and 2) are involved in a Duschinsky rotation with angle $\theta$ (see below for a generalization to more rotated modes);

- we suppose small Duschinsky rotation amplitudes (to first order in $\theta$ ). For SFG, it is possible to explicitly calculate the general case, but it does not lead to a clear separation of the resonant terms. 
We have for the $(\mathrm{N}, \mathrm{N})$ square matrix $\mathbf{R}(\theta)$ describing the rotation:

$$
\mathbf{R}=\left(\begin{array}{cccccc}
\cos \theta & -\sin \theta & & & & \\
\sin \theta & \cos \theta & 0 & \ldots & \ldots & 0 \\
0 & 1 & 0 & \ldots & 0 \\
\ldots & \ldots & \ldots & \ldots & \ldots \\
\ldots & \ldots & \ldots & \ldots & \ldots \\
0 & 0 & \ldots & 0 & 1
\end{array}\right)=\left(\begin{array}{rr}
\mathbf{R}_{\theta} & \mathbf{0}_{2, N-2} \\
\mathbf{0}_{N-2,2} & \mathbf{I d}_{N-2, N-2}
\end{array}\right)
$$

where $\mathbf{R}_{\theta}=\left(\begin{array}{rr}\cos \theta & -\sin \theta \\ \sin \theta & \cos \theta\end{array}\right) \approx\left(\begin{array}{cc}1 & -\theta \\ \theta & 1\end{array}\right)$ and $\mathbf{Q}_{\mathbf{e}}=\mathbf{R} \mathbf{Q}_{\mathbf{g}}+\boldsymbol{\Delta}$.

All matrices $\mathbf{M}$ involved in the calculations have the same form as matrix $\mathbf{R}$ : a $2 \times 2$ submatrix $\mathbf{M}_{\theta}$ for modes 1 and 2, depending on $\theta$, and a $(\mathrm{N}-2) \mathrm{x}(\mathrm{N}-2)$ diagonal submatrix for modes 3 to $\mathrm{N}$, independent on $\theta$ and for which the formulas without mode mixing are still valid.

As shown in the Supplementary Material, we have for IR-resonant SFG

$$
\begin{aligned}
\beta_{i j k}^{S F G}=\frac{-i}{\hbar^{2}} \int_{0}^{\infty} d t e^{i\left(\omega_{S F G}-\omega_{e g}^{0}\right) t-\Gamma_{e g} t} \prod_{j=1}^{N} g_{j}(t) \times \\
\left(\sum_{l=1}^{I R-a c t i v e} \frac{A_{l}^{i j k} \Delta_{l} h_{l}^{D I S}(t)}{\omega_{I R}-\omega_{l}^{g}+i \Gamma_{l}}\left[1-\frac{2}{\hbar} \Delta_{1} \Delta_{2} \theta\left(\omega_{1}^{g}-\omega_{2}^{g}\right) h_{1}^{D I S}(t) h_{2}^{D I S}(t)\right]\right. \\
\left.+\frac{A_{1}^{i j k} \Delta_{2} h_{2}^{D I S}(t) \theta P_{1}(t)}{\omega_{I R}-\omega_{1}^{g}+i \Gamma_{1}}-\frac{A_{2}^{i j k} \Delta_{1} h_{1}^{D I S}(t) \theta P_{2}(t)}{\omega_{I R}-\omega_{2}^{g}+i \Gamma_{2}}\right)
\end{aligned}
$$

with

$$
\begin{aligned}
& P_{1}(t)=\frac{\omega_{2}^{g}}{\omega_{1}^{g}}+h_{1}^{D I S}(t)\left[\frac{\omega_{2}^{g}}{\omega_{1}^{g}}-1\right] \\
& P_{2}(t)=\frac{\omega_{1}^{g}}{\omega_{2}^{g}}+h_{2}^{D I S}(t)\left[\frac{\omega_{1}^{g}}{\omega_{2}^{g}}-1\right]
\end{aligned}
$$

These expressions are valid for arbitrary mode distortions.

In the limit of small frequency shifts, after linearization of all $h_{j}^{D I S}(t)$ as functions of $\lambda_{j}$ (Eq. 20), it is possible to integrate in order to recover the energy denominators and introduce the overlap spectral function $\Phi^{F S}(\omega)$. For an explicit expression of $\beta^{S F G}$, we neglect the higher order terms in $\left(1-\lambda_{j}\right) \theta$ and $\left(1-\lambda_{j}\right)\left(1-\lambda_{k}\right)$ arising from the development of $h_{j}^{D I S}(t)$ in Eq. C3 and use $h_{j}^{D I S}(t) \approx h_{j}^{F S}(t)$ when necessary. 
For example, with

$$
h_{j}^{F S}(t)=-\frac{1}{2}\left[1-e^{-i \omega_{j}^{e} t}\right]=-\frac{1}{2} \sum_{p=0}^{p=1}(-1)^{p} e^{-i p \omega_{j}^{e} t}
$$

we have

$$
\begin{gathered}
h_{1}^{F S}(t) h_{2}^{F S}(t)=\frac{1}{4}\left[1-e^{-i \omega_{1}^{e} t}-e^{-i \omega_{2}^{e} t}+e^{-i\left(\omega_{1}^{e}+\omega_{2}^{e}\right) t}\right] \\
P_{1}(t) h_{2}^{F S}(t)=-\frac{1}{4 \omega_{1}^{g}} \sum_{p=0}^{p=1}(-1)^{p} e^{-i p \omega_{2}^{e} t}\left[\left(\omega_{1}^{g}+\omega_{2}^{g}\right)-\left(\omega_{1}^{g}-\omega_{2}^{g}\right) e^{-i \omega_{1}^{e} t}\right] \\
P_{2}(t) h_{1}^{F S}(t)=-\frac{1}{4 \omega_{2}^{g}} \sum_{p=0}^{p=1}(-1)^{p} e^{-i p \omega_{1}^{e} t}\left[\left(\omega_{1}^{g}+\omega_{2}^{g}\right)-\left(\omega_{2}^{g}-\omega_{1}^{g}\right) e^{-i \omega_{2}^{e} t}\right]
\end{gathered}
$$

leading to

$$
\beta_{i j k}^{S F G}=\frac{1}{\hbar^{2}}\left(\sum_{l}^{I R-a c t i v e} \frac{A_{l}^{i j k} D_{l}^{M I X}\left(\omega_{S F G}\right)}{\omega_{I R}-\omega_{l}^{g}+i \Gamma_{l}^{g}}+\frac{A_{1}^{i j k} D_{1}^{C T}\left(\omega_{S F G}\right)}{\omega_{I R}-\omega_{1}^{g}+i \Gamma_{1}^{g}}+\frac{A_{2}^{i j k} D_{2}^{C T}\left(\omega_{S F G}\right)}{\omega_{I R}-\omega_{2}^{g}+i \Gamma_{2}^{g}}\right)
$$

with

$$
D_{l}^{M I X}(\omega)=D_{l}^{D I S}(\omega)+D_{l}^{\theta}(\omega)
$$

where $D_{l}^{D I S}$ refers to Eq. 26 and

$$
\begin{aligned}
& D_{l}^{\theta}(\omega)=-\frac{\Delta_{l} \Delta_{1} \Delta_{2} \theta\left(\omega_{1}^{g}-\omega_{2}^{g}\right)}{4 \hbar}\left[\Phi^{F S}(\omega)-\Phi^{F S}\left(\omega-\omega_{l}^{e}\right)\right. \\
& -\Phi^{F S}\left(\omega-\omega_{1}^{e}\right)+\Phi^{F S}\left(\omega-\omega_{l}^{e}-\omega_{1}^{e}\right) \\
& -\Phi^{F S}\left(\omega-\omega_{2}^{e}\right)+\Phi^{F S}\left(\omega-\omega_{l}^{e}-\omega_{2}^{e}\right) \\
& \left.+\Phi^{F S}\left(\omega-\omega_{1}^{e}-\omega_{2}^{e}\right)-\Phi^{F S}\left(\omega-\omega_{l}^{e}-\omega_{1}^{e}-\omega_{2}^{e}\right)\right] \\
& D_{1}^{C T}(\omega)=+\frac{\Delta_{2} \theta}{4 \omega_{1}^{g}}\left\{\left(\omega_{1}^{g}+\omega_{2}^{g}\right)\left[\Phi^{F S}(\omega)-\Phi^{F S}\left(\omega-\omega_{2}^{e}\right)\right]\right. \\
& \left.-\left(\omega_{1}^{g}-\omega_{2}^{g}\right)\left[\Phi^{F S}\left(\omega-\omega_{1}^{e}\right)-\Phi^{F S}\left(\omega-\omega_{1}^{e}-\omega_{2}^{e}\right)\right]\right\} \\
& D_{2}^{C T}(\omega)=-\frac{\Delta_{1} \theta}{4 \omega_{2}^{g}}\left\{\left(\omega_{1}^{g}+\omega_{2}^{g}\right)\left[\Phi^{F S}(\omega)-\Phi^{F S}\left(\omega-\omega_{1}^{e}\right)\right]\right. \\
& \left.-\left(\omega_{2}^{g}-\omega_{1}^{g}\right)\left[\Phi^{F S}\left(\omega-\omega_{2}^{e}\right)-\Phi^{F S}\left(\omega-\omega_{1}^{e}-\omega_{2}^{e}\right)\right]\right\}
\end{aligned}
$$


Accordingly, we have for DFG:

$$
\begin{aligned}
\beta_{i j k}^{D F G}=\frac{1}{\hbar^{2}} & \left(\sum_{l}^{I R} \frac{A_{l}^{i j k} D_{l}^{M I X}\left(\omega_{D F G}+\omega_{l}^{g}\right)}{\omega_{I R}-\omega_{l}^{g}-i \Gamma_{l}^{g}}\right. \\
& \left.+\frac{A_{1}^{i j k} D_{1}^{C T}\left(\omega_{D F G}+\omega_{1}^{g}\right)}{\omega_{I R}-\omega_{1}^{g}-i \Gamma_{1}^{g}}+\frac{A_{2}^{i j k} D_{2}^{C T}\left(\omega_{D F G}+\omega_{2}^{g}\right)}{\omega_{I R}-\omega_{2}^{g}-i \Gamma_{2}^{g}}\right)
\end{aligned}
$$

These expressions may be easily computed and used for fitting procedures of experimental curves. Except for extreme cases (i.e. large coefficients $S_{j}$ ), the development of the overlap

spectral function as a function of $k_{j}$ may be truncated to a low value of $\sum_{j} k_{j}$, thus reducing the calculation times for the expressions above.

Finally, we consider the extension to the case where $\mathrm{P}$ vibration modes in the excited state are involved in a small amplitude Duschinsky rotation by pair, i.e. $(1,2) ;(3,4) ; \ldots$; $(\mathrm{P}-1, \mathrm{P})$ with $\mathrm{P} \leq \mathrm{N}$. In this situation, all matrices above are block diagonal and may be decomposed into $\mathrm{P} / 2(2 \times 2)$ blocks and a diagonal matrix. Repeating the previous analysis block by block, with the help of the properties of block diagonal matrices, we find that, at first order in all angles of mixing, the equations C10 and C15 must simply be modified by the addition of terms analogous to the $(1,2)$ terms: one $D^{\theta}$ for each pair in $D_{l}^{M I X}(\omega)$, together with a total of $\mathrm{P}$ distinct $D^{C T}$ terms.

\section{REFERENCES}

${ }^{1}$ B. Bozzini, B. Busson, G. P. De Gaudenzi, C. Mele, and A. Tadjeddine, J. Solid State Electrochem. 12, 303 (2008).

${ }^{2}$ A. Le Rille and A. Tadjeddine, J. Electroanal. Chem. 467, 238 (1999).

${ }^{3}$ H. Arnolds, Prog. Surf. Sci. 86, 1 (2011).

${ }^{4}$ E. H. G. Backus, A. Eichler, A. W. Kleyn, and M. Bonn, Science 310, 1790 (2005).

${ }^{5}$ L. Dalstein, A. Revel, C. Humbert, and B. Busson, J. Chem. Phys. 148, 134701 (2018).

${ }^{6}$ G. Rupprechter, Catal. Today 126, 3 (2007).

${ }^{7}$ P. E. Ohno, H.-f. Wang, and F. M. Geiger, Nat. Commun. 8, 1032 (2017).

${ }^{8}$ C.-S. Hsieh, R. K. Campen, M. Okuno, E. H. G. Backus, Y. Nagata, and M. Bonn, Proc. Natl. Acad. Sci. U. S. A. 110, 18780 (2013).

${ }^{9}$ R. Khatib, E. H. G. Backus, M. Bonn, M.-J. Perez-Haro, M.-P. Gaigeot, and M. Sulpizi, Sci. Rep. 6, 24287 (2016). 
${ }^{10}$ D. Hu, A. Mafi, and K. C. Chou, J. Phys. Chem. B 120, 2257 (2016).

${ }^{11}$ L. Dalstein, C. Humbert, M. Ben Haddada, S. Boujday, G. Barbillon, and B. Busson, J. Phys. Chem. Lett. 10, 7706 (2019).

${ }^{12}$ N. Alyabyeva, A. Ouvrard, A.-M. Zakaria, and B. Bourguignon, J. Phys. Chem. Lett. 10, 624 (2019).

${ }^{13}$ S. H. Lin and A. A. Villaeys, Phys. Rev. A 50, 5134 (1994).

${ }^{14}$ B. Busson and A. Tadjeddine, J. Phys. Chem. C 113, 21895 (2009).

${ }^{15}$ M. Sovago, E. Vartiainen, and M. Bonn, J. Phys. Chem. C 113, 6100 (2009).

${ }^{16}$ J. Wang, Z. Paszti, M. L. Clarke, X. Chen, and Z. Chen, J. Phys. Chem. B 111, 6088 (2007).

${ }^{17}$ S. Lin, M. Hayashi, R. Islampour, J. Yu, D. Yang, and G. Y. Wu, Phys. B Condens. Matter 222, 191 (1996).

${ }^{18}$ C. Humbert, B. Busson, C. Six, A. Gayral, M. Gruselle, F. Villain, and A. Tadjeddine, J. Electroanal. Chem. 621, 314 (2008).

${ }^{19}$ C. Humbert, L. Dreesen, S. Nihonyanagi, T. Masuda, T. Kondo, A. Mani, K. Uosaki, P. Thiry, and A. Peremans, Appl. Surf. Sci. 212-213, 797 (2003).

${ }^{20}$ L. Dreesen, C. Humbert, Y. Sartenaer, Y. Caudano, C. Volcke, A. A. Mani, A. Peremans, P. A. Thiry, S. Hanique, and J.-M. Frère, Langmuir 20, 7201 (2004).

${ }^{21}$ Y. Caudano, C. Silien, C. Humbert, L. Dreesen, A. Mani, A. Peremans, and P. Thiry, J. Electron Spectros. Relat. Phenomena 129, 139 (2003).

${ }^{22}$ M. Raschke, M. Hayashi, S. Lin, and Y. Shen, Chem. Phys. Lett. 359, 367 (2002).

${ }^{23}$ K. C. Chou, S. Westerberg, Y. R. Shen, P. N. Ross, and G. A. Somorjai, Phys. Rev. B 69, 153413 (2004).

${ }^{24}$ S. Sengupta, L. Bromley III, and L. Velarde, J. Phys. Chem. C 121, 3424 (2017).

${ }^{25}$ D. Elsenbeck, S. K. Das, and L. Velarde, Phys. Chem. Chem. Phys. 19, 18519 (2017).

${ }^{26}$ S. K. Das, S. Sengupta, and L. Velarde, J. Phys. Chem. Lett. 7, 320 (2016).

${ }^{27}$ M. Raab, J. C. Becca, J. Heo, C.-K. Lim, A. Baev, L. Jensen, P. N. Prasad, and L. Velarde, J. Chem. Phys. 150, 114704 (2019).

${ }^{28}$ R.-h. Zheng, W.-m. Wei, Y.-y. Jing, H. Liu, and Q. Shi, J. Phys. Chem. C 117, 11117 (2013).

${ }^{29}$ T. Nagahara, H. Fukushima, M. Aida, and T.-a. Ishibashi, Chem. Phys. Lett. 506, 190 (2011). 
${ }^{30}$ T. Miyamae, Y. Miyata, and H. Kataura, J. Phys. Chem. C 113, 15314 (2009).

${ }^{31}$ T. Miyamae, K. Tsukagoshi, and W. Mizutani, Phys. Chem. Chem. Phys. 12, 14666 (2010).

${ }^{32}$ S. Yang, H. Noguchi, and K. Uosaki, J. Phys. Chem. C 119, 26056 (2015).

${ }^{33}$ M. Okuno, D. Ishikawa, W. Nakanishi, K. Ariga, and T.-a. Ishibashi, J. Phys. Chem. C 121, 11241 (2017).

${ }^{34}$ Q. Li, R. Hua, and K. C. Chou, J. Phys. Chem. B 112, 2315 (2008).

${ }^{35}$ G. Herzberg, Krieger Publ. Co. Malabar, FL, USA (Krieger Publishing Company: Malabar, FL, USA, 1991).

${ }^{36}$ J. Tang, Chem. Phys. 188, 143 (1994).

${ }^{37}$ J. Tang, M. T. Lee, and S. H. Lin, J. Chem. Phys. 119, 7188 (2003).

${ }^{38}$ J. Vallet, A. Boeglin, J. Lavoine, and A. Villaeys, Chem. Phys. Lett. 241, 203 (1995).

${ }^{39}$ J. C. Vallet, A. J. Boeglin, J. P. Lavoine, and A. A. Villaeys, Phys. Rev. A 53, 4508 (1996).

${ }^{40}$ T. E. Sharp and H. M. Rosenstock, J. Chem. Phys. 41, 3453 (1964).

${ }^{41}$ K.-M. Chen and C.-C. Pei, Chem. Phys. Lett. 165, 523 (1990).

${ }^{42}$ H. M. Lu and J. B. Page, J. Chem. Phys. 90, 5315 (1989).

${ }^{43}$ V. Hizhyakov and I. Tehver, Phys. status solidi 21, 755 (1967).

${ }^{44}$ C. K. Chan and J. B. Page, J. Chem. Phys. 79, 5234 (1983).

${ }^{45}$ S.-Y. Lee and E. J. Heller, J. Chem. Phys. 71, 4777 (1979).

${ }^{46}$ D. J. Tannor and E. J. Heller, J. Chem. Phys. 77, 202 (1982).

${ }^{47}$ E. J. Heller, R. Sundberg, and D. Tannor, J. Phys. Chem. 86, 1822 (1982).

${ }^{48}$ A. Baiardi, J. Bloino, and V. Barone, J. Chem. Theory Comput. 9, 4097 (2013).

${ }^{49}$ D. S. Egolf, M. R. Waterland, and A. Myers Kelley, J. Phys. Chem. B 104, 10727 (2000).

${ }^{50}$ J. Neugebauer and B. A. Hess, J. Chem. Phys. 120, 11564 (2004).

${ }^{51}$ E. Doktorov, I. Malkin, and V. Man’ko, J. Mol. Spectrosc. 64, 302 (1977).

${ }^{52}$ R. Berger, C. Fischer, and M. Klessinger, J. Phys. Chem. A 102, 7157 (1998).

${ }^{53}$ H. Kupka and P. H. Cribb, J. Chem. Phys. 85, 1303 (1986).

${ }^{54}$ R. Islampour, M. Hayashi, and S. Lin, Chem. Phys. Lett. 234, 7 (1995).

${ }^{55}$ A. Warshel and P. Dauber, J. Chem. Phys. 66, 5477 (1977).

${ }^{56}$ D. L. Tonks and J. B. Page, J. Chem. Phys. 88, 738 (1988).

${ }^{57}$ Y. J. Yan and S. Mukamel, J. Chem. Phys. 85, 5908 (1986). 
${ }^{58}$ C. K. Chan, J. Chem. Phys. 81, 1614 (1984).

${ }^{59}$ B. R. Stallard, P. M. Champion, P. R. Callis, and A. C. Albrecht, J. Chem. Phys. 78, $712(1983)$.

${ }^{60}$ S. A. Lee, C. K. Chan, J. B. Page, and C. T. Walker, J. Chem. Phys. 84, 2497 (1986).

${ }^{61}$ T. W. Patapoff, P. Y. Turpin, and W. L. Peticolas, J. Phys. Chem. 90, 2347 (1986).

${ }^{62}$ Y. Fujimura and S. H. Lin, J. Chem. Phys. 70, 247 (1979).

${ }^{63}$ H. Lu and J. B. Page, Chem. Phys. Lett. 131, 87 (1986).

${ }^{64}$ H. M. Lu and J. B. Page, J. Chem. Phys. 88, 3508 (1988).

${ }^{65}$ C. S. Reddy and M. D. Prasad, J. Phys. Chem. A 120, 2583 (2016).

${ }^{66}$ T. Petrenko and F. Neese, J. Chem. Phys. 127, 164319 (2007).

${ }^{67}$ R. Borrelli, A. Capobianco, and A. Peluso, J. Phys. Chem. A 116, 9934 (2012).

${ }^{68}$ J. Y. Huang and Y. R. Shen, Phys. Rev. A 49, 3973 (1994).

${ }^{69}$ M. Hayashi, S. H. Lin, M. B. Raschke, and Y. R. Shen, J. Phys. Chem. A 106, 2271 (2002).

${ }^{70}$ P. A. Weiss, D. W. Silverstein, and L. Jensen, J. Phys. Chem. Lett. 5, 329 (2014).

${ }^{71}$ M. A. Belkin, Y. R. Shen, and R. A. Harris, J. Chem. Phys. 120, 10118 (2004).

${ }^{72}$ M. Hayashi, S. H. Lin, and Y. R. Shen, J. Phys. Chem. A 108, 8058 (2004).

${ }^{73}$ T. I. Rush and W. L. Peticolas, J. Phys. Chem. 99, 14647 (1995).

${ }^{74}$ W. L. Peticolas and T. Rush, J. Comput. Chem. 16, 1261 (1995).

${ }^{75}$ R. Kumble, T. S. Rush, M. E. Blackwood Jr, P. M. Kozlowski, and T. G. Spiro, J. Phys. Chem. B 102, 7280 (1998).

${ }^{76}$ R.-h. Zheng, D.-m. Chen, W.-m. Wei, T.-j. He, and F.-c. Liu, J. Phys. Chem. B 110, 4480 (2006).

${ }^{77}$ J. Guthmuller and B. Champagne, J. Chem. Phys. 127, 164507 (2007).

${ }^{78}$ J. Guthmuller and B. Champagne, J. Phys. Chem. A 112, 3215 (2008).

${ }^{79}$ J. Guthmuller and B. Champagne, ChemPhysChem 9, 1667 (2008).

${ }^{80}$ S. Shim, C. M. Stuart, and R. A. Mathies, ChemPhysChem 9, 697 (2008).

${ }^{81}$ L. Jensen, L. L. Zhao, J. Autschbach, and G. C. Schatz, J. Chem. Phys. 123, 174110 (2005).

${ }^{82}$ L. Jensen and G. C. Schatz, J. Phys. Chem. A 110, 5973 (2006).

${ }^{83}$ F. Santoro, A. Lami, R. Improta, J. Bloino, and V. Barone, J. Chem. Phys. 128, 224311 (2008). 
${ }^{84}$ F. Santoro, A. Lami, R. Improta, and V. Barone, J. Chem. Phys. 126, 184102 (2007).

${ }^{85}$ R. Zaleśny, W. Bartkowiak, and B. Champagne, Chem. Phys. Lett. 380, 549 (2003).

${ }^{86}$ J. B. Page and D. L. Tonks, J. Chem. Phys. 75, 5694 (1981).

${ }^{87}$ K. Huang and A. Rhys, Proc. R. Soc. London. Ser. A. Math. Phys. Sci. 204, 406 (1950).

${ }^{88}$ C. Chan and J. Page, Chem. Phys. Lett. 104, 609 (1984).

${ }^{89}$ D. L. Tonks and J. B. Page, Chem. Phys. Lett. 79, 247 (1981).

90Z. F. Deng and S. Mukamel, J. Chem. Phys. 85, 1738 (1986).

${ }^{91}$ C. G. Tetsassi Feugmo, V. Liégeois, Y. Caudano, F. Cecchet, and B. Champagne, J. Chem. Phys. 150, 074703 (2019).

${ }^{92}$ D. W. Silverstein and L. Jensen, J. Chem. Phys. 136, 064110 (2012).

${ }^{93}$ D. W. Silverstein and L. Jensen, J. Chem. Phys. 136, 064111 (2012). 